\title{
Design and Analysis of a Magnetic Bearings with Three Degrees of Freedom
}

\author{
Ye Yuan ${ }^{1}$, Yukun Sun ${ }^{2 *}$ and Qianwen Xiang
}

\begin{abstract}
The current research of supporting and transmission system in flywheel energy storage system (FESS) focuses on the low consumption design. However, friction loss is a non-negligible factor in the high-speed but lightweight FESS energy and momentum storage with mechanical-type supporting system. In order to realize the support system without mechanical loss and to maximize the efficiency of the flywheel battery, a permanent magnet biased magnetic bearings (PMBMB) is applied to the FESS with the advantages of low loss, high critical speed, flexible controllability and compact structure. In this frame, the relevant research of three degrees of freedom (3-DOF) PMBMB for a new type FESS is carried out around the working principle, structural composition, coupling characteristics analysis, mathematical model, and structural design. In order to verify the performance of the 3-DOF PMBMB, the radial force mathematical model and the coupling determination equations of radial two DOF are calculated according to an equivalent magnetic circuit, and radial-axial coupling is analyzed through finite element analysis. Moreover, a control system is presented to solve the control problems in practical applications. The rotor returns to the balanced position in $0.05 \mathrm{~s}$ and maintains stable suspension. The displacement fluctuation is approximately $40 \mu \mathrm{m}$ in the $y$ direction and $30 \mu \mathrm{m}$ in the $x$ direction. Test results indicate that the dynamic rotor of the proposed flywheel energy storage system with PMBMB has excellent characteristics, such as good start-of-suspension performance and stable suspension characteristics. The proposed research provides the instruction to design and control a low loss support system for FESS.
\end{abstract}

Keywords: Flywheel energy storage system, Magnetic bearings, Degrees of freedom, Coupling, Finite element analysis

\section{Introduction}

The year 2015 was an extraordinary one for renewable energy, with the largest global capacity additions seen to date [1]. The year saw several developments including a dramatic decline in global fossil fuel prices; a series of announcements regarding the lowest-ever prices for renewable power long-term contracts; a significant increase in attention to energy storage; and a historic climate agreement in Paris that brought together the global community. Renewables are now established around the world as mainstream sources of energy [2, 3]. Rapid growth, particularly in the power sector, is driven by several factors, including the improving cost-competiveness

\footnotetext{
*Correspondence: sykujs421@163.com

${ }^{2}$ College of Electrical Engineering, Nanjing Institute of Technology, Nanjing 211100, China

Full list of author information is available at the end of the article
}

of renewable technologies, dedicated policy initiatives, better access to financing, energy security and environmental concerns, growing demand for energy in developing and emerging economies, and the need for access to modern energy [4]. However, the challenges still remain, particularly beyond the power sector. The development of renewable energy technologies can be used to relieve or solve the dependence on traditional energy sources. Before the renewable energy technologies are widely applied, it is necessary to develop the energy storage technology to improve energy efficiency especially in the fields of renewable energy, distributed power generation, electric vehicles, and so on.

Flywheel energy storage system (FESS) is an energy storage device [5] with long life, high energy storage density, and low rotational loss; thus, it is considered the most competitive and promising energy storage technology and has received attention from scholars recently $[6$, 
7]. FESS stores mechanical energy [8] that is converted into electrical energy in a manner analogous to that of electrochemical batteries [9] with chemical energy. The materials for the flywheel, the type of electrical machine, the type of bearings, and the confinement atmosphere determine the energy efficiency [10] (>85\%) of flywheelbased energy storage systems. A flywheel system stores mechanical energy that interchanges in the form of electrical energy by means of an electrical machine/generator with a bidirectional power converter.

For fully integrated flywheel battery, compact structure, low scattering and high efficiency are the main consideration of design objectives. Friction loss is a non-negligible factor in the high-speed but lightweight flywheel energy storage system (FESS) for energy and momentum storage with mechanical-type supporting system $[11,12]$. In order to realize the support system without mechanical loss $[13,14]$, to maximize the efficiency of the flywheel battery [15], permanent magnet biased magnetic bearings (PMBMB) is applied to the FESS with the advantages of low loss, high critical speed [16], flexible controllability and compact structure [17]. Compared with the active magnetic bearing, the heat loss is smaller, the structure is more compact, and the reliability is higher in FESS with the PMBMB supporting system $[18,19]$.

The flywheel rotor in FESS is rotated by an electric machine operating as a motor at electric energy storage mode. In the case of a breakdown of electric current, the flywheel rotor rotated by its inertia under vacuum conditions makes the electric machine operate as a generator [20]. As the core of an energy conversion unit, the type of motor/generator utilized for high-power FESS exerts a significant effect on the overall performance of an energy storage device [21]. Reliable, fail-safe, robust, compact, and low-cost electric motors are needed for FESS with high temperatures or extreme temperature variations. The bearingless switched reluctance motor (BSRM) [22, 23] possesses these characteristics. Given that the structure of $\mathrm{MB}$ is similar to that of the stator of a switched reluctance motor (SRM), bearing-less technology is applied to SRM to fully enhance its high-speed performance $[24,25]$. BSRM can not only rotate but also realize two DOF levitation $[26,27]$ by integrating a magnetic levitation winding into the motor stator. Conventional $\mathrm{MB}$ are a source of energy losses and require lubrication to relieve mechanical friction. High rotational speed of the flywheel rotor in motoring mode generally leads to large friction loss because of wearing. A solution is to select a three DOF hybrid magnetic bearings (HMB) [28] with low suspension power consumption.

In this study, a permanent magnet biased $M B$ (PMBMB) that is among the most recommended HMB for reducing cost and consumption is utilized as a three
DOF MB that can produce a biased magnetic field with minimal winding. The rotor system is frequently supported by DC active magnetic bearings and a magnetized permanent magnet. Power amplifiers (e.g., linear and switching power amplifiers) can drive a DC magnetic bearing. Thus, a five DOF levitation system can be realized with PMBMB and BSRM. This study focuses on a theory of design and analysis for a three DOF PMBMB. First, the working principle of PMBMB is introduced, and a method of structural parameter design is proposed. Second, the radial force mathematical model and the coupling determination equations of radial two DOF are derived based on an equivalent magnetic circuit. Finally, radial-axial coupling is analyzed through finite element analysis, and the theoretical analyses are verified based on the experimental results of the test facility.

\section{New Type of FESS}

One of the key technologies of FESS is its supporting technique, which can not only support the operation of the flywheel (making it operate stably) but also reduce the size of the flywheel rotor. At present, the types of supporting parts are mechanical, superconducting $\mathrm{MB}$, electromagnetic suspension, and permanent $\mathrm{MB}$ support. Figure 1 shows a new type of FESS with the characteristics of short axial length, compact structure, flexible control strategy, and minimal loss.

The new FESS mainly consists of a bearing-less motor, a three DOF PMBMB, and an auxiliary bearing. The bearing-less motor is a single-winding bearing-less flywheel motor (SWBFM) [29] with the advantages of low loss, high specific power and fault-tolerant performance which is improved from the structure of BSRM.

Figure 2(a) shows the configuration of a three DOF PMBMB (the axial control coils are hidden in active

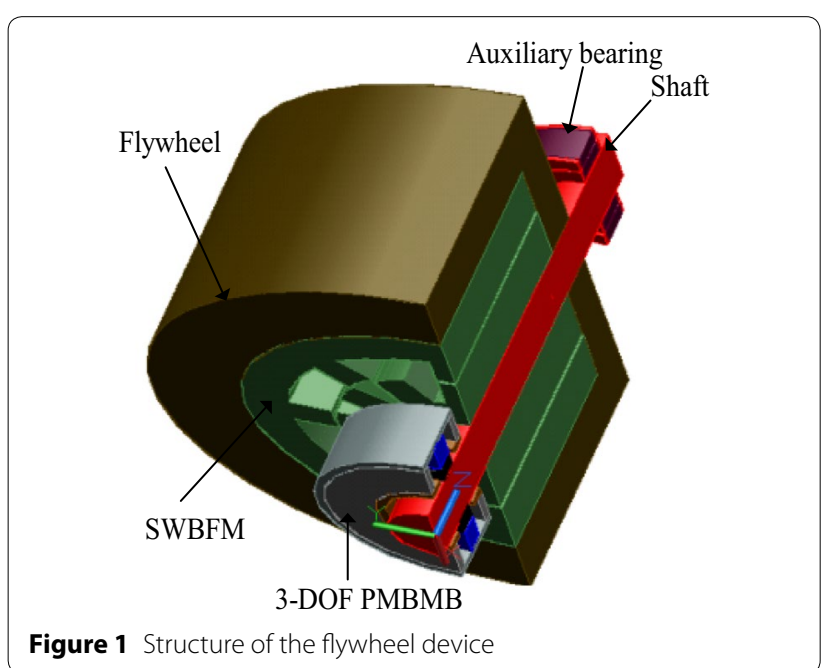




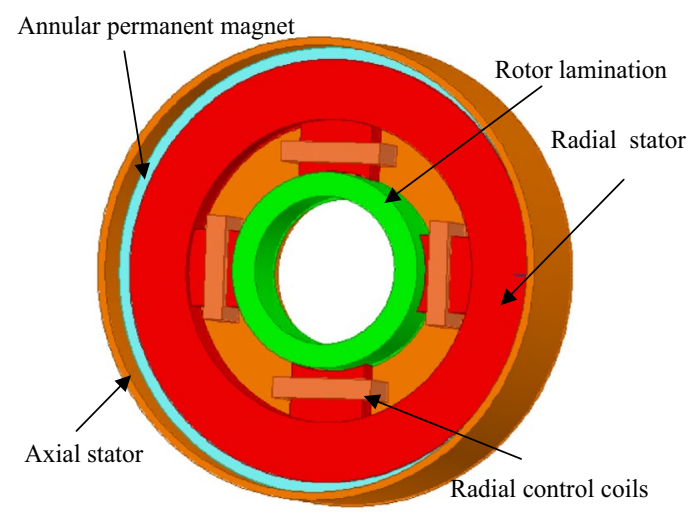

a Configuration of a three DOF PMBMB

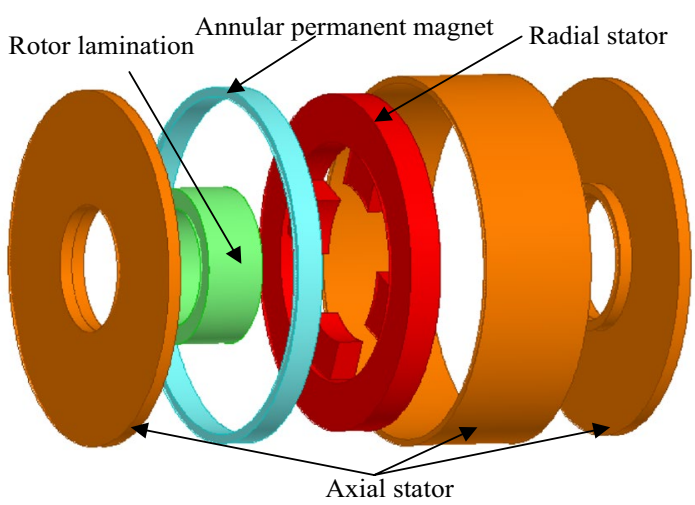

b Magnified view of a three DOF PMBMB

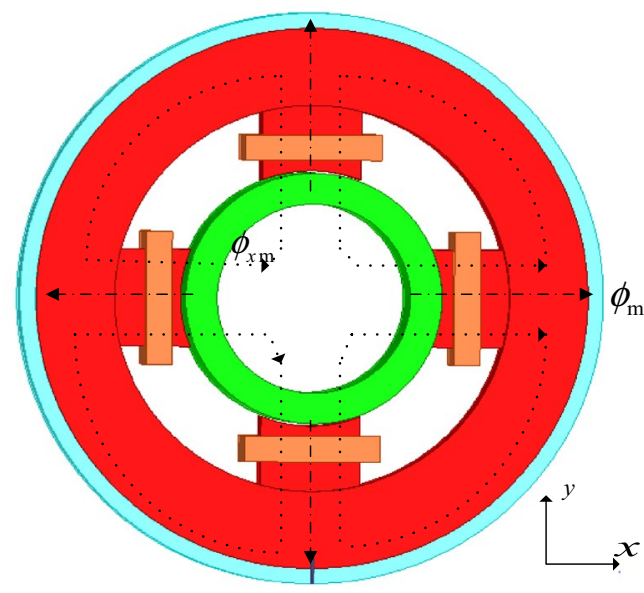

C Schematic of radial force

Figure 2 Schematics of a three DOF PMBMB

view), and its magnified view is shown in Figure 2(b) (axial and radial control coils are hidden in active view). The PMBMB consists of a radial stator with four poles, four radial control coils, a rotor laminator, an axial stator with two radial control coils, and an axial magnetized permanent magnet (PM).

If the rotor is suspended in an equilibrium position, only biased flux is generated by the PM circuits in MB. Once the rotor diverges from the equilibrium position, control flux is added to biased flux. Consequently, static biased and control fluxes are synthesized through addition and subtraction. The resultant force generated by the synthesized magnetic flux suspends the rotor in the equilibrium position.

The operation principle of the radial force of the 3-DOF PMBMB is introduced in Figure 2(c). The $\phi_{x \mathrm{~m}}$ produced by radial control coils is the control magnetic flux in the $x$-direction, and $\phi_{\mathrm{m}}$ is the bias magnetic flux produced by PM.

Given the symmetrical structure of PMBMB, $\phi_{\mathrm{m}}$ is a constant in the positive and negative $x$-directions. If the rotor is pushed in the negative $x$-direction by an external force, then the length of the air gap increases and $\phi_{x \mathrm{~m}}$ decreases in the positive $x$-direction. The length of the air gap decreases when $\phi_{\mathrm{m}}$ increases in the negative $x$-direction. $\phi_{\mathrm{m}}$ can be produced by radial control coils to strengthen the $\phi_{\mathrm{m}}$ in the positive $x$-direction and balance the magnetic flux.

According to the symmetric structure of PMBMB, $\phi_{\mathrm{m}}$ is a constant in $x$ positive and $x$ negative directions. If the rotor lamination moves toward the $x$ negative direction because of a external force acting on it, $\phi_{\mathrm{m}}$ would decrease in the $x$ positive direction because of the increased length of air gap. Similarly, $\phi_{\mathrm{m}}$ increases in the $x$ negative direction. $\phi_{x \mathrm{~m}}$ can be produced by radial control coils to strengthen the $\phi_{\mathrm{m}}$ in the $x$ positive direction to balance the magnetic flux in the $x$ negative direction.

\section{Parameter Design of PMBMB}

A method for the parameter design of PMBMB was developed. The design goal is that the maximum value of axial levitation force is $200 \mathrm{~N}$, whereas the maximum value of radial levitation force is $100 \mathrm{~N}$. The inside diameter of the rotor lamination is $37 \mathrm{~mm}$, the outer diameter is $49 \mathrm{~mm}$, and the axial length is $18 \mathrm{~mm}$. The structure of the rotor lamination is shown in Figure 3, where $r_{\mathrm{i}}$ is the inside diameter, $r_{\mathrm{j}}$ is the outer diameter, and $l$ is the axial length. $S_{z}$ is defined as the sectional area of the axial magnetic pole, and $S_{x y}$ is defined as the sectional area of the radial magnetic pole $\left(r_{\mathrm{i}}, r_{\mathrm{j}}\right.$, and $l$ are regarded as given variables).

\subsection{Calculation of Equivalent Magnetic Circuit for PMBMB}

The equivalent magnetic circuit of PMBMB is shown in Figure 4. $G_{z 1}$ and $G_{z 2}$ are defined as the magnetic conductance of the axial air gap, $N_{z}$ is defined as the 


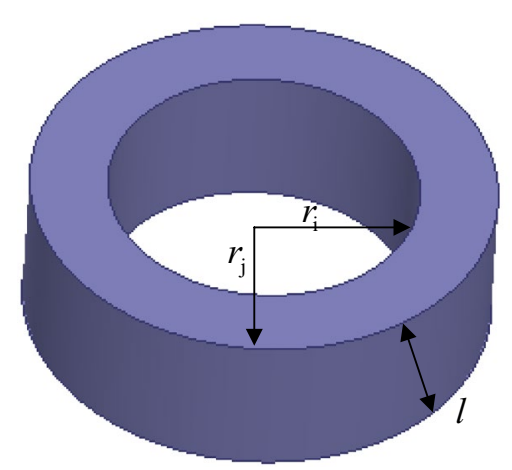

Figure 3 Rotor lamination of PMBMB

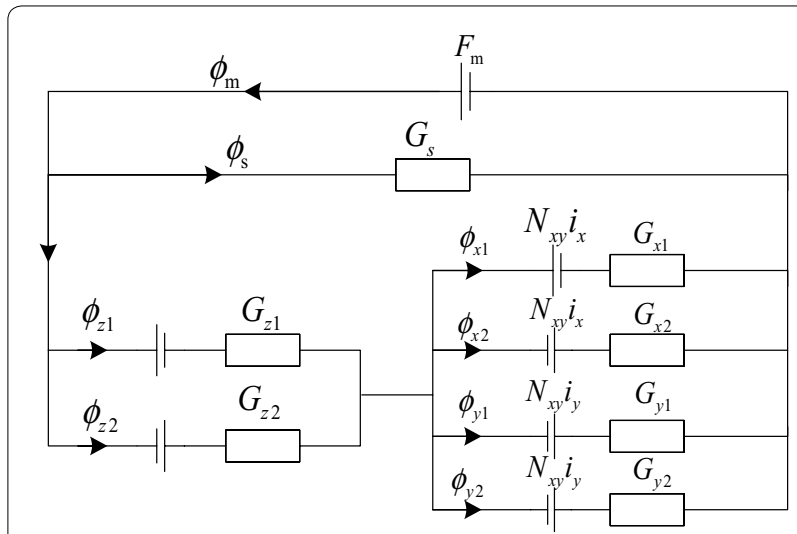

Figure 4 Equivalent hybrid excitation magnetic circuit of PMBMB

number of the axial control coils, and $i_{z}$ is defined as the current of the axial control coils. $G_{x 1}, G_{x 2}, G_{y 1}$, and $G_{y 2}$ are defined as the magnetic conductance of the radial air gap, and $N_{x y}$ is defined as the number of radial control coils. $i_{x}$ and $i_{y}$ are the current of the radial control coils. $\phi_{\mathrm{s}}$ is the leakage magnetic flux, and $G_{\mathrm{s}}$ is the leakage magnetic conductance. $F_{\mathrm{m}}$ is the magnetomotive force produced by PM. The relationship among $G_{x 1}$, $G_{x 2}, G_{y 1}, G_{y 2}, G_{z 1}$, and $G_{z 2}$ can be expressed as follows:

$$
\left\{\begin{array}{l}
G_{x}=G_{x 1}+G_{x 2}, \\
G_{y}=G_{y 1}+G_{y 2}, \\
G_{z}=G_{z 1}+G_{z 2}, \\
G_{g}=G_{x}+G_{y}+G_{z} .
\end{array}\right.
$$

In a balanced position, $g_{z}$ is defined as the axial length of the air gap, and $g_{x y}$ is defined as the radial length of the air gap. $x$ and $y$ are the values of displacement in the radial direction, and $z$ is the value of displacement in the axial direction. Thus, magnetic conductance can be written as follows:

$$
\left\{\begin{array}{l}
G_{x 1}=\frac{\mu_{0} S_{x y}}{g_{x y}-x}, \quad G_{y 1}=\frac{\mu_{0} S_{x y}}{g_{x y}-y}, \\
G_{x 2}=\frac{\mu_{0} S_{x y}}{g_{x y}+x}, \quad G_{z 1}=\frac{\mu_{0} S_{z}}{g_{z}-z}, \\
G_{y 2}=\frac{\mu_{0} S_{x y}}{g_{x y}+y}, \quad G_{z 2}=\frac{\mu_{0} S_{z}}{g_{z}+z} .
\end{array}\right.
$$

The magnetic flux can be obtained as

$$
\begin{aligned}
\phi_{x 1}= & {\left[F_{\mathrm{m}} G_{z}-N_{z} i_{z}\left(G_{z 1}-G_{z 2}\right)-N_{x y} i_{y}\left(G_{y 2}-\right.\right.} \\
& \left.\left.G_{y 1}\right)-N_{x y} i_{x}\left(G_{z}+2 G_{x 2}+G_{y}\right)\right] /\left[G_{\mathrm{g}} / G_{x 1}\right],
\end{aligned}
$$

$$
\begin{aligned}
\phi_{x 2}= & {\left[F_{\mathrm{m}} G_{z}-N_{z} i_{z}\left(G_{z 1}-G_{z 2}\right)-N_{x y} i_{y}\left(G_{y 2}-\right.\right.} \\
& \left.\left.G_{y 1}\right)+N_{x y} i_{x}\left(G_{z}+2 G_{x 1}+G_{y}\right)\right] /\left[G_{\mathrm{g}} / G_{x 2}\right],
\end{aligned}
$$

$$
\begin{aligned}
\phi_{y 1}= & {\left[F_{\mathrm{m}} G_{z}-N_{z} i_{z}\left(G_{z 1}-G_{z 2}\right)-N_{x y} i_{x}\left(G_{x 2}-\right.\right.} \\
& \left.\left.G_{x 1}\right)-N_{x y} i_{y}\left(G_{z}+2 G_{y 2}+G_{x}\right)\right] /\left[G_{\mathrm{g}} / G_{y 1}\right],
\end{aligned}
$$

$$
\begin{aligned}
\phi_{y 2}= & {\left[F_{\mathrm{m}} G_{z}-N_{z} i_{z}\left(G_{z 1}-G_{z 2}\right)-N_{x y} i_{x}\left(G_{x 2}-\right.\right.} \\
& \left.\left.G_{x 1}\right)+N_{x y} i_{y}\left(G_{z}+2 G_{y 1}+G_{x}\right)\right] /\left[G_{\mathrm{g}} / G_{y 2}\right],
\end{aligned}
$$

$$
\begin{aligned}
& \phi_{z 1}= {\left[F_{\mathrm{m}}\left(G_{x}+G_{y}\right)-N_{z} i_{z}\left(G_{x}+2 G_{z 2}+G_{y}\right)+\right.} \\
&\left.N_{x y} i_{x}\left(G_{x 2}-G_{x 1}\right)-N_{x y} i_{y}\left(G_{y 2}-G_{y 1}\right)\right] /\left[G_{\mathrm{g}} / G_{z 1}\right], \\
& \phi_{z 2}= {\left[N_{z} i_{z}\left(G_{x}+2 G_{z 1}+G_{y}\right)-N_{x y} i_{y}\left(G_{y 2}-G_{y 1}\right)\right.} \\
&+\left.F_{\mathrm{m}}\left(G_{x}+G_{y}\right)+N_{x y} i_{x}\left(G_{x 2}-G_{x 1}\right)\right] /\left[G_{\mathrm{g}} / G_{z 2}\right] .
\end{aligned}
$$

$F_{x}$ is defined as the levitation force in the $x$ direction, $F_{y}$ is levitation force in the $y$ direction, and $F_{z}$ is levitation force in the $z$ direction (axial direction). Thus, $F_{x}, F_{y}$, and $F_{z}$ can be expressed as

$$
\left\{\begin{array}{l}
F_{x}=\frac{\phi_{x 1}^{2}-\phi_{x 2}^{2}}{2 \mu_{0} S_{x y}} \\
F_{y}=\frac{\phi_{y 1}^{2}-\phi_{y 2}^{2}}{2 \mu_{0} S_{x y}} \\
F_{z}=\frac{\phi_{z 1}^{2}-\phi_{z 2}^{2}}{2 \mu_{0} S_{z}}
\end{array}\right.
$$

When the rotor lamination is at the balanced position, the magnetic induction intensity on one side of the PMBMB should be increased to the maximum value, defined as $B_{\mathrm{s}}$, to produce the maximum value of levitation force. Correspondingly, the magnetic induction intensity on the other side of the PMBMB should be decreased to zero. In this study, the value of $B_{\mathrm{s}}$ is $1.2 \mathrm{~T}$. Thus, the maximum value of levitation force can be derived as 


$$
\left\{\begin{array}{l}
2 F_{x y \mathrm{~m}}=\frac{B_{s}^{2} S_{z}}{2 \mu_{0}}, \\
F_{z \mathrm{~m}}=\frac{B_{s}^{2} S_{z}}{2 \mu_{0}}, \\
F_{x y \mathrm{~m}}=\frac{B_{s}^{2} S_{x y}}{2 \mu_{0}} .
\end{array}\right.
$$

The results of Eq. (10) are $S_{z}=350 \mathrm{~mm}^{2}$ and $S_{x y}=175$ $\mathrm{mm}^{2}$.

\subsection{Design of Radial and Axial Poles}

The design goal is as follows: the duty ratio of radial poles is 0.5 , defined as $f$; the number of radial poles is 4 ; the length of air gap is $0.5 \mathrm{~mm}$; and the maximum value of radial levitation force is $100 \mathrm{~N} . b_{\mathrm{p}}, h_{\mathrm{p}}$, and $l_{1}$ are the width, length, and height of the radial pole, respectively. $t_{\mathrm{p}}$ corresponds to the arc length. The relationship of structure size is expressed as

$$
\left\{\begin{array}{l}
f=\frac{n t_{\mathrm{p}}}{2 \pi\left(r_{\mathrm{p}}+g_{1}\right)}, \\
b_{\mathrm{p}}=2\left(r+g_{1}\right) \sin \frac{t_{\mathrm{p}}}{2\left(r+g_{1}\right)} .
\end{array}\right.
$$

Thus,

$$
\left\{\begin{array}{l}
b_{\mathrm{p}}=2\left(r+g_{1}\right) \sin \frac{f \pi}{n}, \\
S_{x y}=\varphi_{1} b_{\mathrm{p}} l_{1}, h_{\mathrm{p}}=\frac{2 S_{x y}}{b_{\mathrm{p}} \varphi_{2}},
\end{array}\right.
$$

where the value of $\phi_{1}$ is 0.9 and the value of $\phi_{2}$ is 0.6.

From Figure 5, the structure parameters can be derived as $R_{1}=22.5 \mathrm{~mm}, R_{2}=20 \mathrm{~mm}, d_{1}=d_{3}=4 \mathrm{~mm}$, and $d_{2}=2$ $\mathrm{mm}$.

\subsection{Parameter Design of PM}

For an annular PM that is radially magnetized, $D_{\mathrm{p}}$ is the inside diameter, $T_{\mathrm{p}}(3 \mathrm{~mm})$ is the magnetization thickness, and $L_{\mathrm{p}}$ is the axial length. When $h_{\mathrm{p}}$ is derived, $D_{\mathrm{p}}$ is also derived. $B_{\mathrm{p}}$ and $H_{\mathrm{p}}$ are the parameters of the working point according to the $B-H$ curve. The relationship between $B_{\mathrm{p}}$ and $H_{\mathrm{p}}$ can be expressed as

$$
B_{\mathrm{p}}=-\mu_{\mathrm{p}} H_{\mathrm{p}}+B_{\mathrm{r}},
$$

where $\mu_{\mathrm{p}}$ is the magnetic permeability and $\mu_{\mathrm{p}}=B_{\mathrm{r}} / H$. $B_{\mathrm{r}}$ is the residual flux density.

$$
\left\{\begin{array}{l}
H_{\mathrm{p}} T_{\mathrm{p}}=\frac{2 B_{0} g_{0}}{\mu_{0}}, B_{0}=\frac{B_{\mathrm{s}}}{2}, \\
S_{\mathrm{p}}=\frac{\phi_{\mathrm{m}}}{B_{\mathrm{p}}}, \quad L_{\mathrm{p}}=\frac{S_{\mathrm{p}}}{\pi\left(D_{1 \mathrm{p}}+\frac{T_{\mathrm{p}}}{2}\right)},
\end{array}\right.
$$

where $S_{\mathrm{p}}$ is the cross-sectional area of PM. Thus, the value of $L_{\mathrm{p}}$ is $8 \mathrm{~mm}$. The number of windings can be written as follows:

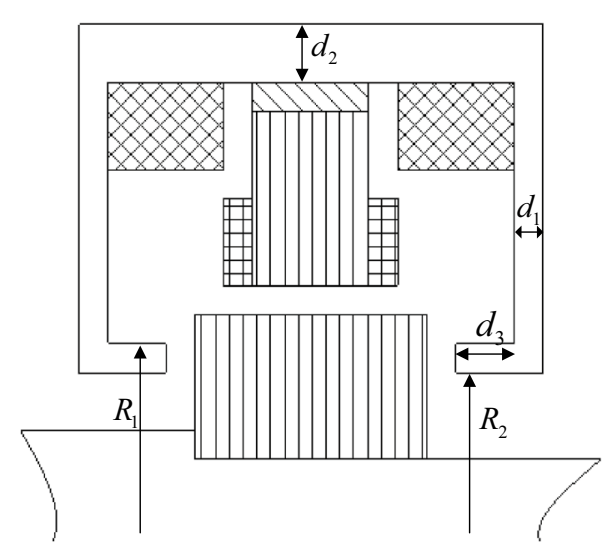

Figure 5 Structure of the axial stator

$$
N i=\frac{B_{0} g_{0}}{\mu_{0}} .
$$

Hence, the value of $N_{x y}$ and $N_{z}$ is 120 , and the rated current is $4 \mathrm{~A}$.

\subsection{Finite Element Analysis of PMBMB}

The finite element model of PMBMB was analyzed with Ansoft. The magnetic flux density distribution of the radial stator and rotor is shown in Figure 6.

The magnetic flux density distribution when the rotor is at the balanced position and the value of $i$ is zero is shown in Figure 6(a). The bias magnetic field line produced by PM is a closed loop in radial poles, RL, and air gap. The magnetic flux density distribution in the $x$ and $y$ directions is symmetrical. Thus, the resultant force is zero.

The magnetic flux density distribution when the rotor is at the balanced position and the value of $i$ is maximum (4 A) is shown in Figure 6(b). The radial levitation force in $x$ positive $(108 \mathrm{~N})$ and $y$ negative directions $(-110 \mathrm{~N})$ can be produced without magnetic circuit saturation. The value of $F_{x}$ is $108 \mathrm{~N}$, the value of $F_{y}$ is $-110 \mathrm{~N}$, and the value of $F_{z}$ is $204 \mathrm{~N}$, which meet the design requirements.

\section{Coupling Analysis of PMBMB}

The analysis of PMBMB focused on radial two-DOF coupling and radial-axial coupling.

\subsection{Numerical Analysis for Radial Two-DOF Coupling}

The air gap reluctance is less than the circular PM reluctance, and the control magnet flux produced by radial coil does not pass through the PM and the magnetic circuit of 


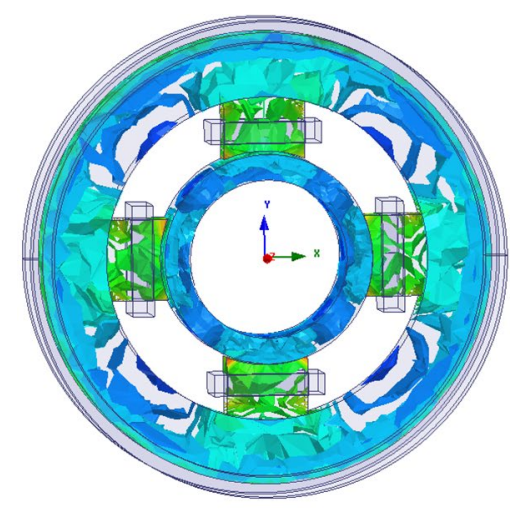

a The value of $i$ is zero

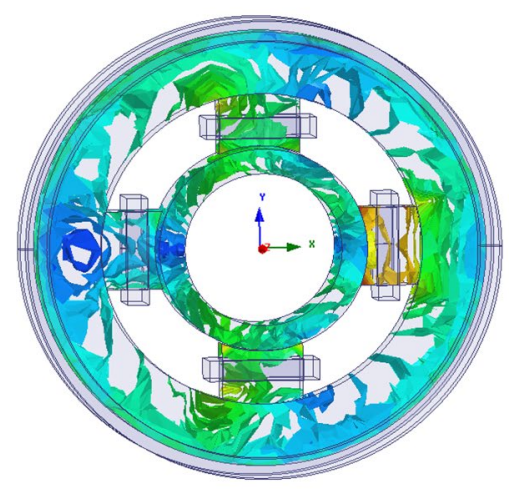

b The value of $i$ is maximum

Figure 6 Magnetic flux density distribution of the radial stator and rotor lamination

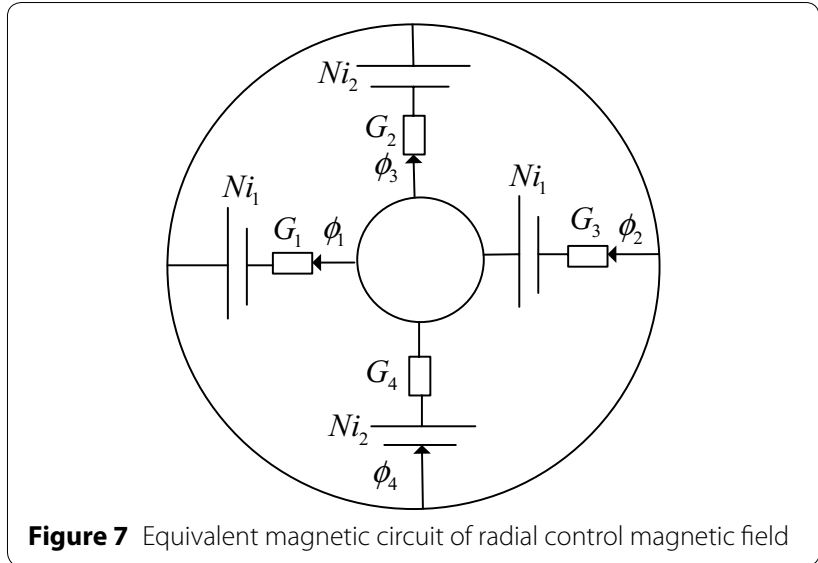

magnetic flux leakage. The equivalent magnetic circuit of the radial control magnetic field is shown in Figure 7. The $i_{1}$ and $i_{2}$ are the current of radial coils.

According to the loop and node equations, the magnetic flux is as follows:

$$
\left\{\begin{array}{l}
\phi_{1}=\frac{N i_{1}\left(G_{2}+2 G_{3}+G_{4}\right)+N i_{2}\left(-G_{4}+G_{2}\right)}{1+\frac{G_{2}}{G_{1}}+\frac{G_{3}}{G_{1}}+\frac{G_{4}}{G_{1}}}, \\
\phi_{2}=\frac{N i_{1}\left(G_{1}-G_{3}\right)+N i_{2}\left(2 G_{4}+G_{1}+G_{3}\right)}{1+\frac{G_{1}}{G_{2}}+\frac{G_{3}}{G_{2}}+\frac{G_{4}}{G_{2}}}, \\
\phi_{3}=\frac{N i_{1}\left(2 G_{1}+G_{2}+G_{4}\right)+N i_{2}\left(G_{4}-G_{2}\right)}{1+\frac{G_{1}}{G_{3}}+G_{2}}, G_{3}+G_{4} \\
\phi_{4}=\frac{N i_{1}\left(G_{3}-G_{1}\right)+N i_{2}\left(2 G_{2}+G_{1}+2 G_{2}\right)}{1+\frac{G_{1}}{G_{4}}+\frac{G_{2}}{G_{4}}+\frac{G_{3}}{G_{4}}} .
\end{array}\right.
$$

In extreme cases, the eccentric displacement of the rotor is approximately $1 / 10$ of the air gap length:

$$
\left\{\begin{array}{l}
G_{1}=G_{2}=11 G_{x y} / 10 \\
G_{3}=G_{4}=11 G_{x y} / 10
\end{array}\right.
$$

where $G_{x y}$ is the magnetic conductance of the radial air gap in the balanced position. The coupling determination equations of radial two DOFs are

$$
\left\{\begin{array}{l}
\phi_{1}=0.96 N i_{1} G_{x y}-0.046 N i_{2} G_{x y}, \\
\phi_{2}=0.96 N i_{2} G_{x y}-0.046 N i_{1} G_{x y} \\
\phi_{3}=1.1 N i_{1} G_{x y}+0.06 N i_{2} G_{x y} \\
\phi_{4}=0.06 N i_{1} G_{x y}+1.1 N i_{2} G_{x y} .
\end{array}\right.
$$

According to Eq. (18), the magnetic flux of the air gap in the $x$ direction produced by the control coil of the $y$ direction is approximately $1 / 20$ of the magnetic flux produced by the control coil of the $x$ direction. Similarly, the magnetic flux of the air gap in the $y$ direction produced by the control coil of the $x$ direction is approximately $1 / 20$ of the magnetic flux produced by the control coil of the $y$ direction. Thus, the mutual coupling of magnetic flux in the radial two DOFs ( $x$ and $y$ directions) can be ignored.

\subsection{Finite Element Analysis of Radial-Axial Coupling}

The relationship of radial-axial coupling is present in Figure 8. The relationship among radial control current $i_{x}$, displacement in the $z$ direction, and radial levitation force $F_{x}$ is shown in Figure 8(a). The rotor is at the balanced position in $x$ and $y$ directions, and the value of control current in $x$ and $y$ directions is zero. Figure 8(a) indicates that when the axial displacement in the $z$ direction fluctuates within $[-0.2 \mathrm{~mm}, 0.2 \mathrm{~mm}]$, almost no influence is exerted on $F_{x}$ because of the axial displacement. However, the edge area is nonlinear. Accordingly, the current stiffness coefficient is not the same in the levitating stage and at the balanced position.

The relationship among radial control current $i_{z}$, displacement in the $x$ direction, and radial levitation force $F_{x}$ is shown in Figure 8(b). The rotor is at the balanced position in $z$ and $y$ directions, and the value of control 


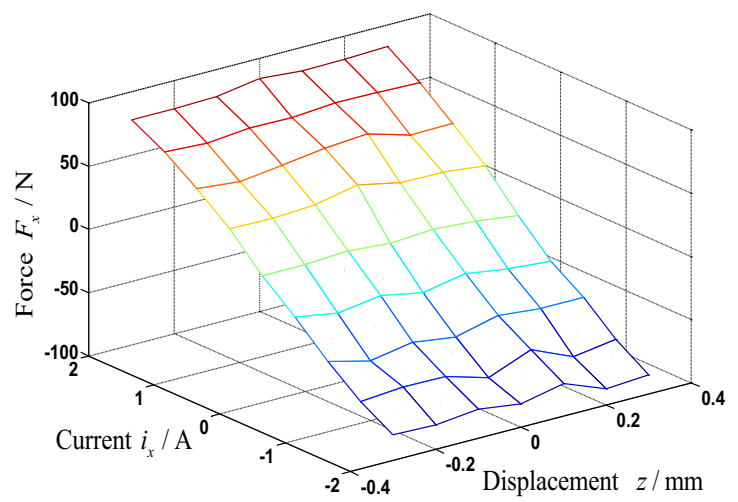

a Relationship among $i_{x}, z$, and $F_{x}$

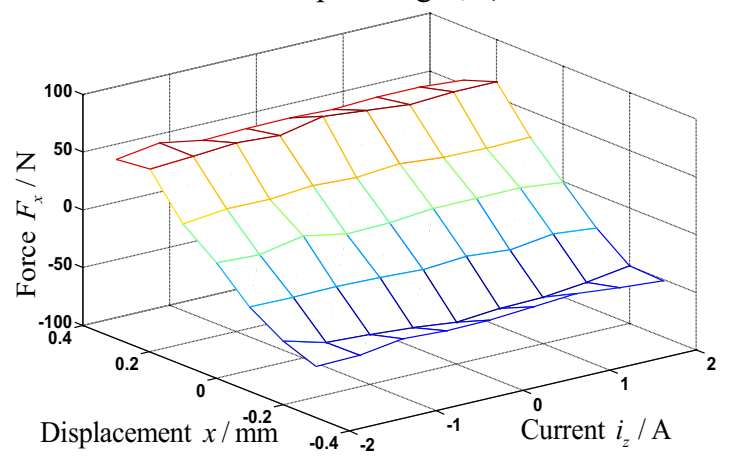

b Relationship among $i_{z}, z$, and $F_{x}$

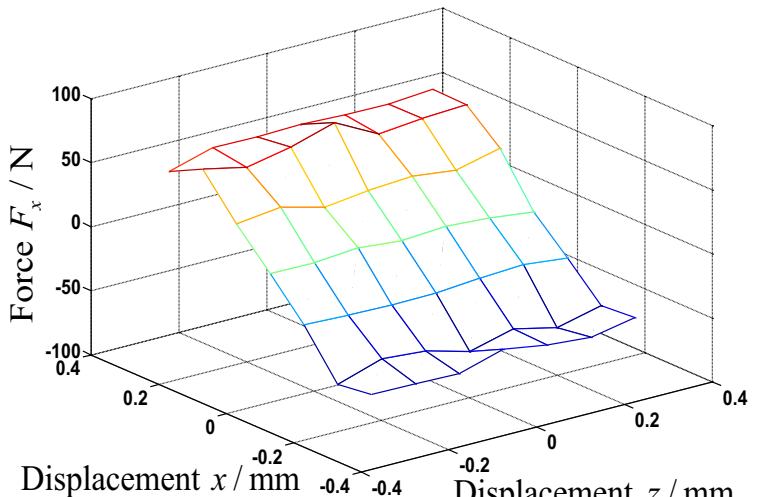

Displacement $x / \mathrm{mm} \quad-0.4 \quad-0.4 \quad$ Displacement $z / \mathrm{mm}$

C Relationship among $x, z$, and $F_{x}$

Figure 8 Relationship among forces, currents, and displacements

current in $x$ and $y$ directions is zero. Figure 8(b) shows that (1) when radial displacement in the $x$ direction fluctuates within $[-0.2 \mathrm{~mm}, 0.2 \mathrm{~mm}]$, almost no influence is exerted on $F_{x}$ because of the control current in $z$ direction; (2) when radial displacement in the $x$ direction fluctuates within $[-0.3 \mathrm{~mm},-0.2 \mathrm{~mm}]$ and $[0.2 \mathrm{~mm}, 0.3$ $\mathrm{mm}$ ], nonlinear areas appear according to the magnetic saturation with a small length of air gap. Comparison of
Figures 8(a) and 10(b) indicates that control current has more influence on radial levitation force than the displacement of displacement in the $z$ direction.

Figure 8(c) shows that (1) when radial displacement in the $x$ direction fluctuates within $[-0.2 \mathrm{~mm}, 0.2 \mathrm{~mm}$, the graph tilts at a certain angle with the $x-y$ plane. The radial levitation force is thus proportional to radial displacement, and axial displacement has minimal influence on radial levitation force; (2) when radial displacement in the $x$ direction fluctuates within [ $-0.3 \mathrm{~mm},-0.2 \mathrm{~mm}$ ] and $[0.2 \mathrm{~mm}, 0.3 \mathrm{~mm}]$, the corresponding area appears nonlinear, which indicates that coupling occurs between axial and radial control in the rotor-floating stage.

The relationship among radial control current $i_{x}$, eccentricity in the $x$-direction, and radial levitation force $F_{x}$ is exhibited in Figure 9. The rotor is at the balanced position in the $z$-and $y$-directions, and the value of control current in the $z$-and $y$-directions is zero. When the radial eccentricity in the $x$-direction fluctuates at $[-0.2 \mathrm{~mm}$, $0.2 \mathrm{~mm}$ ], the graph tilts at a certain angle along the $x-y$ plane. The relationship between radial eccentricity $x$, and radial levitation force $F_{x}$ is exhibited in Figure 4(a). When the radial eccentricity in the $x$-direction fluctuates at $[-0.2 \mathrm{~mm}, 0.2 \mathrm{~mm}]$, the radial levitation force is $F_{x}$ proportional to $x$. When the radial eccentricity in the $x$-direction fluctuates at $[-0.2 \mathrm{~mm},-0.4 \mathrm{~mm}]$ and at [0.2 $\mathrm{mm}, 0.4 \mathrm{~mm}]$, the relationship between $x$, and $F_{x}$ is nonlinear. The relationship between radial control current $i_{x}$, and radial levitation force $F_{x}$ is exhibited in Figure 4 (b). When the radial eccentricity in the $x$-direction fluctuates at $\left[-2 \mathrm{~A}, 2 \mathrm{~A}\right.$ ], the radial levitation force is $F_{x}$ proportional to radial control current $i_{x}$. When the radial eccentricity in the $x$-direction fluctuates at $[-4 \mathrm{~A},-2 \mathrm{~A}]$ and at $[2 \mathrm{~A}, 4 \mathrm{~A}]$, the relationship between $i_{x}$, and $F_{x}$ is nonlinear.

\section{Experiments for Floating and Displacement}

The block diagram of the control system for the threeDOF PMBMB is presented in Figure 10. The control system for the three-DOF PMBMB includes an external displacement closed-looped control and an internal current closed-looped control. The differential forward proportional-integral-derivative (PID) control method is adopted in the displacement closed-looped control. A comparison method of hysteretic current is adopted in the current closed-looped control.

The feedback signals of $x, y$, and $z$ positions, which are processed in an interface circuit board, can be obtained when the rotor is displaced from the balanced position by external disturbance. The position deviation signals (i.e., the difference between the balanced position reference signals and the position feedback signals) are then sent to the PID controllers. The PID controllers transform the 


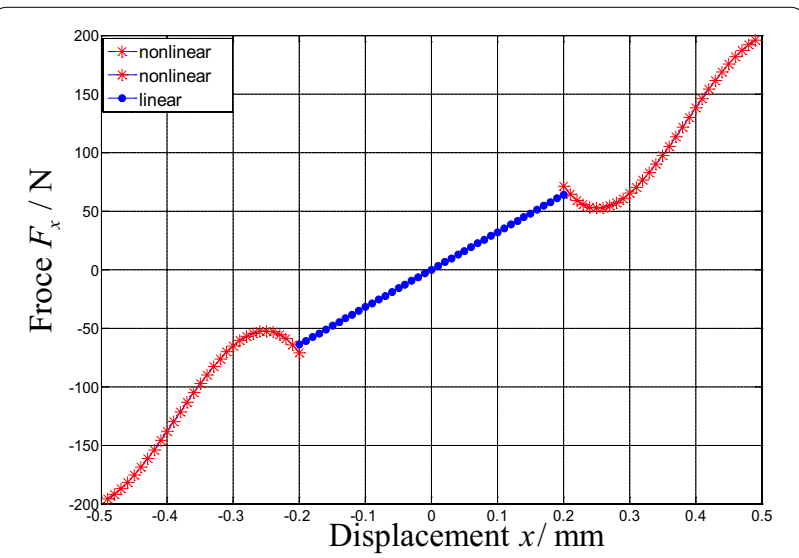

a Relationship between $x$ and $F_{x}$

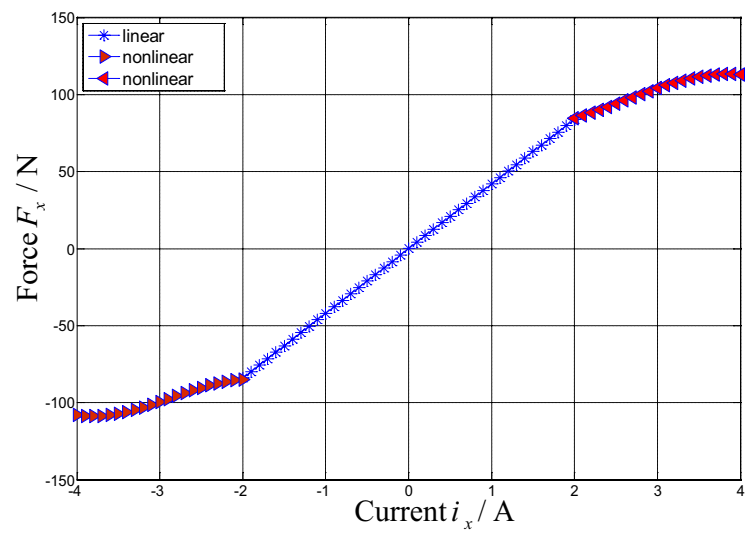

b Relationship between $i_{x}$ and $F_{x}$

Figure 9 Relationship between $x, i_{x}$ and $F_{x}$

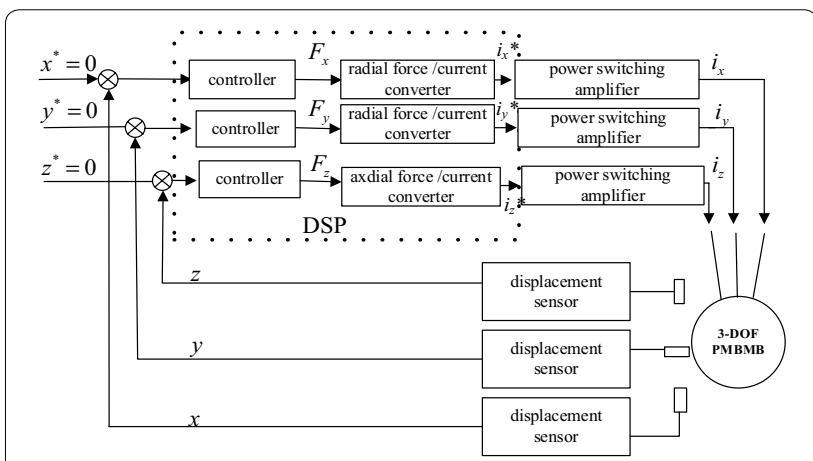

Figure 10 Block diagram of the control system for PMBMB

deviation signals into force signals $\left(F_{x}, F_{y}\right.$, and $\left.F_{z}\right)$ that are outputted to the force-current transformation modules. The current reference signals $\left(i_{\mathcal{x}}^{*} i_{y}\right.$, and $\left.i_{z}\right)$ are obtained from the force-current transformation modules. The excitation currents $\left(i_{x}, i_{y}\right.$, and $\left.i_{z}\right)$ to the radial and axial control coils can be obtained through the switching power amplifiers. Through this process, the control currents are adjusted by the control system with a negative position and current feedbacks to readjust the flux distribution. In addition, the rotor can be suspended in the ideal balanced position. If the rotor is suspended in the balanced position (i.e., $x^{*}=0, y^{*}=0$, and $z^{*}=0$ ), the output of the PID controllers will be zero because the displacement deviation is zero (i.e., the exciting current will also be zero), and the rotor can be suspended by the PM.

A PID controller with a differential restriction link and integral separation is applied in the control system. The transfer function of this controller is obtained as follows:

$$
G(s)=\left\{\begin{array}{l}
\frac{K_{\mathrm{p}}\left(1+T_{\mathrm{d}} S\right)}{\left(1+\tau T_{\mathrm{d}} S\right)}\left(1+\frac{1}{T_{\mathrm{i}} S}\right),|e| \leq c, \\
\frac{K_{\mathrm{p}}\left(1+T_{\mathrm{d}} S\right)}{\left(1+\tau T_{\mathrm{d}} S\right)},|e|>c,
\end{array}\right.
$$

where $K_{\mathrm{p}}$ is the proportionality coefficient, $T_{\mathrm{i}}$ is the integral action time constant, $T_{\mathrm{d}}$ is the derivative action time constant, $\tau$ is the differential gain coefficient, $e$ is the difference between the equilibrium position reference signals and the position feedback signals, and $c$ is the threshold value.

In this study, the experimental system of PMBMB was designed according to the specification shown in Table 1 to achieve stable suspension. The experimental waveforms are shown in Figure 11.

Figure 11(a) shows the floating waveform of displacement in the $x$ direction. The rotor remains at the stationary position $(0.2 \mathrm{~mm}, 0.25 \mathrm{~mm})$ before floating to the balanced position. After conducting the control coil, the rotor returns to the balanced position in $0.05 \mathrm{~s}$ and maintains stable suspension.

Figure 11(b) shows the displacement waveforms at $n=1200 \mathrm{r} / \mathrm{min}$ in $x$ and $y$ directions. When the rotor is in the stable suspension stage, the unilateral displacement fluctuation in the $y$ direction is approximately $40 \mu \mathrm{m}$, which is $8 \%$ of the length of the air gap; the unilateral displacement fluctuation in the $x$ direction is approximately $30 \mu \mathrm{m}$, which is also $8 \%$ of the length of the air gap. The displacement fluctuation is significantly less than the length of the air gap. The PMBMB designed in this study therefore has excellent properties.

\section{Conclusions}

In order to realize the support system without mechanical loss and to maximize the efficiency of the flywheel battery, a PMBMB is applied to the new FESS with the advantages of low loss, high critical speed, flexible controllability and compact structure. The research is focus on the design and analysis of the PMBMB. 
Table 1 Specification of a prototype

\begin{tabular}{ll}
\hline Parameter & Value \\
\hline Axial maximum capacity $F_{z}(\mathrm{~N})$ & 200 \\
Raidial maximum capacity $F_{x y}(\mathrm{~N})$ & 100 \\
Maximum current $i(\mathrm{~A})$ & 4 \\
Number of winding turns & 120 \\
Length of air gap $g(\mathrm{~mm})$ & 0.5 \\
Axial magnetic pole area $\mathrm{S}_{z}\left(\mathrm{~mm}^{2}\right)$ & 350 \\
Radial magnetic pole area $\mathrm{S}_{x y}\left(\mathrm{~mm}^{2}\right)$ & 175 \\
\hline
\end{tabular}

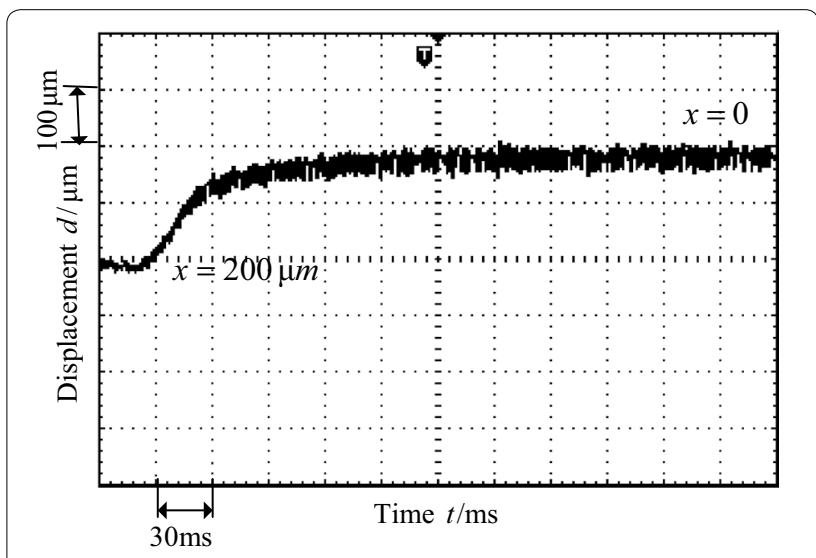

a Floating waveform

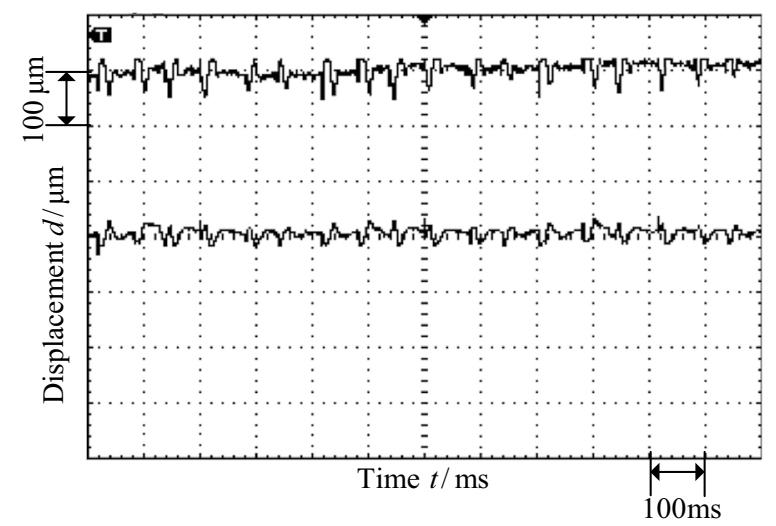

b Displacement waveforms at $n=1200 \mathrm{r} / \mathrm{min}$

Figure 11 Experimental waveforms of PMBMB

(1) The parameter design method of PMBMB is obtained and verified by the finite element analysis.

(2) A control system design of a three-DOF PMBMB is designed according to the numerical analysis and finite element analysis of radial-axial coupling. The theory results show that the coupling near the equilibrium position can be ignored.

(3) The performance experiments show that the designed PMBMB has excellent properties of floating and stable suspension.

\section{Authors' Contributions}

YS was in charge of the whole trial; YY wrote the manuscript; QX assisted with sampling and laboratory analyses. All authors read and approved the final manuscript.

\section{Author Details}

${ }^{1}$ School of Electrical and Information Engineering, Jiangsu University, Zhenjiang 212013, China. ${ }^{2}$ College of Electrical Engineering, Nanjing Institute of Technology, Nanjing 211100, China.

\section{Authors' Information}

Ye Yuan, born in 1991, is currently a lecturer at School of Electrical and Information Engineering, Jiangsu University, China. His research interests include design and control of bearingless motors, low loss flywheel batteries supporting and drive system.

Yukun Sun, born in 1958, is currently a professor at College of Electrical Engineering, Nanjing Institute of Technology, China. His research interests include intelligent control of special electric power transmission, power quality control, intelligent detection and control of microbial environment.

Qianwen Xiang, born in 1982, is currently an associate professor at School of Electrical and Information Engineering, Jiangsu University, China. Her research interests include optimization design of bearingless motor, intelligent control of special electrical machine.

\section{Competing interests}

The authors declare no competing financial interests.

\section{Funding}

Supported by National Natural Science Foundation of China (Grant Nos. 51707082, 51877101, 51607080), Jiangsu Provincial Natural Science Foundation of China (Grant Nos. BK20170546, BK20150510), China Postdoctoral Science Foundation (Grant No. 2017M620192), and Priority Academic Program Development of Jiangsu Higher Education Institutions.

\section{Publisher's Note}

Springer Nature remains neutral with regard to jurisdictional claims in published maps and institutional affiliations.

Received: 22 May 2017 Accepted: 10 January 2019

Published online: 23 January 2019

\section{References}

[1] A Maroosi, A Ahmadi, A E Nezhad, et al. Comment on "resource scheduling under uncertainty in a smart grid with renewables and plug-in vehicles" by AY Saber and GK Venayagamoorthy. IEEE Systems Journal, 2016, 10(1): 147-150.

[2] L Jia, L Tong. Renewables and storage in distribution systems: Centralized vs. decentralized integration. IEEE Journal on Selected Areas in Communications, 2016, 34(3): 665-674.

[3] Y Yuan, Y Sun, Y Huang. Design and analysis of bearingless flywheel motor especially for flywheel energy storage. Electronics Letters, 2016, 52 (1): 66-68.

[4] T Ackermann, E M Carlini, B Ernst, et al. Integrating variable renewables in Europe: current status and recent extreme events. IEEE Power and Energy Magazine, 2015, 13(6): 67-77.

[5] A Kailasan, T Dimond, P Allaire, et al. Design and analysis of a unique energy storage flywheel system - An integrated flywheel, motor/ 
generator, and magnetic bearing configuration. Journal of Engineering for Gas Turbines \& Power, 2015, 137(4): 042505.

[6] M Dowlatshahi, S M Saghaiannejad, JW Ahn, et al. Copper loss and torque ripple minimization in switched reluctance motors considering nonlinear and magnetic saturation effects. Journal of Power Electronics, 2014, 14(2): 351-361.

[7] M D Choudhury, F Ahmed, G Kumar, et al. Design methodology for a special single winding based bearingless switched reluctance motor. The Journal of Engineering, 2017, 1(1): 10.1049/joe.2016.0368.

[8] A Doria-Cerezo. Comments on "Control and performance of a doubly-fed induction machine intended for a flywheel energy storage system". IEEE Transactions on Power Electronics, 2013, 28(1): 605-606.

[9] K Yamomoto, Y Iriyama. Visualization of electrochemical reactions in all-solid-state lid-ion batteries by spatially resolved electron energy-loss spectroscopy and electron holography. Materials Transactions, 2015, 56(5): 617-624.

[10] S Jiang, H Wang, S Wen. Flywheel energy storage system with a permanent magnet bearing and a pair of hybrid ceramic ball bearings. Journal of Mechanical Science \& Technology, 2014, 28(12): 5043-5053.

[11] C Zhang, K J Tseng. Design and control of a novel flywheel energy storage system assisted by hybrid mechanical-magnetic bearings. Mechatronics, 2013, 23(3): 297-309.

[12] H S Zad, TI Khan, I Lazoglu. Design and adaptive sliding mode control of hybrid magnetic bearings. IEEE Transactions on Industrial Electronics, 2017, (99): 1-1.

[13] A Noshadi, J Shi, W S Lee, et al. System identification and robust control of multi-input multi-output active magnetic bearing systems. IEEE Transactions on Control Systems Technology, 2016, 24(4): 1227-1239.

[14] J Sun, Z Ju, C Peng, et al. A novel 4-DOF hybrid magnetic bearing for DGMSCMG. IEEE Transactions on Industrial Electronics, 2017, 64(3): 2196-2204.

[15] B Han, S Zheng, Y Le, et al. Modeling and analysis of coupling performance between passive magnetic bearing and hybrid magnetic radial bearing for magnetically suspended flywheel. IEEE Transactions on Magnetics, 2013, 49(10): 5356-5370.

[16] KWang, D Wang, $\mathrm{H}$ Lin, et al. Analytical modeling of permanent magnet biased axial magnetic bearing with multiple air gaps. IEEE Transactions on Magnetics, 2014, 50(11): 10.1109/TMAG.2014.2330843.
[17] X Cao, H Yang, Z Deng, et al. Equivalent transformation and control for single-winding bearingless switched reluctance motors. Electric Machines \& Power Systems, 2016, 44(9): 1040-1050.

[18] H Wang, Y Wang, X Liu. Design of novel bearingless switched reluctance motor. IET Electr. Power Appl., 2012, 6(2):73-81.

[19] Z Xu, F Zhang, D H Lee, et al. Design and analysis of novel 12/14 hybrid pole type bearingless switched reluctance motor with short flux path. Journal of Electrical Engineering \& Technology, 2012, 7(5):705-713.

[20] S Liu, DY Sun, DT Qin. Analyzing on nonlinear transmission energy flows and loss for the wet axle in the heavy vehicle. Journal of Mechanical Engineering, 2015, 51(9): 10-17. (in Chinese)

[21] W Polkowski, P Jóźwik, R Łyszkowski. Effect of hot differential speed rolling on microstructure and mechanical properties of Fe3Al-based intermetallic alloy. International Journal of Materials Research, 2016, 107(9): 867-871.

[22] HWang, J Liu, J Bao, et al. A novel bearingless switched reluctance motor with a biased permanent magnet. IEEE Transactions on Industrial Electronics, 2014, 61(12): 6947-6955.

[23] Z Liu, Z Deng, X Cao, et al. Design of a full-period bearingless switched reluctance generator. Proceedings of the CSEE, 2011, 31(12):77-83.

[24] ZXu, F Zhang. Design and analysis of a novel 12/14 hybrid pole type bearingless switched reluctance motor. Industrial Electronics (ISIE), 2012 IEEE International Symposium on. IEEE, 2012: 1922-1927.

[25] X Cao, J Zhou and C Liu. Advanced control method for single-winding bearingless switched reluctance motor to reduce torque ripple and radial displacement. IEEE Transactions on Energy Conversion, 2017, (99): 1-1.

[26] HWang, J Bao, B Xue, et al. Control of suspending force in novel permanent-magnet-biased bearingless switched reluctance motor. IEEE Transactions on Industrial Electronics, 2015, 62(7): 4298-4306.

[27] LChen, W Hofmann. Speed regulation technique of one bearingless $8 / 6$ switched reluctance motor with simpler single winding structure. IEEE Transactions on Industrial Electronics, 2012, 59(6): 2592-2600.

[28] B Han, X Liu, S Zheng. A novel integral 5-DOFs hybrid magnetic bearing with one permanent magnet ring used for turboexpander. Mathematical Problems in Engineering, 2014(4): 1-18.

[29] Y Yuan, Y K Sun, Y H Huang. Radial force dynamic current compensation method of single winding bearingless flywheel motor. IET Power Electronics, 2015, 8(7): 1224-1229.

\section{Submit your manuscript to a SpringerOpen ${ }^{\circ}$ journal and benefit from:}

- Convenient online submission

- Rigorous peer review

- Open access: articles freely available online

- High visibility within the field

- Retaining the copyright to your article

Submit your next manuscript at $\boldsymbol{\nabla}$ springeropen.com 